\title{
Un Procedimiento de Evaluación de las Condiciones Necesarias para Innovar la Gestión de Mantenimiento
}

\author{
Fernando F. Espinosa ${ }^{1}$, Acires Dias $^{2}$ y Nelson Back ${ }^{2}$ \\ (1) Universidad de Talca, 2 Norte 685, Talca-Chile (e-mail: fespinos@utalca.cl) \\ (2) Universidad Federal de Santa Catarina, Casilla Postal 476, Campus Universitário, Trindade, \\ 88040-900 Florianópolis,SC-Brasil (e-mail: acires@emc.ufsc.br, backnb@terra.com.br)
}

\begin{abstract}
Resumen
Se presenta un procedimiento estructurado, implementado en Excel ${ }^{\circledR}$, para analizar la situación actual de una empresa con el fin de evaluar las condiciones necesarias para enfrentar un proceso de innovación en el sistema de gestión del mantenimiento. El procedimiento permite obtener una base de conocimientos del sistema técnico de la empresa y de la gestión actual del mantenimiento. El procedimiento está compuesto de una auditoria y un análisis experto basado en reglas que abarcan aspectos relacionados con la forma de gestión, el nivel tecnológico de los equipos productivos, el grado de madurez de la organización, las metas para el mantenimiento y los requerimientos físicos y financieros para implementar las concepciones seleccionadas. Este procedimiento permite identificar las debilidades y fortalezas del sistema productivo y del mantenimiento para planificar en forma óptima el proceso de innovación de la gestión del mantenimiento.
\end{abstract}

\section{A Procedure to Evaluate the Necessary Conditions to Innovate the Maintenance Management}

\begin{abstract}
A structured procedure, implemented in Excel ${ }^{\circledR}$, to analyze the present situation in an enterprise with the purpose of evaluating the necessary conditions to implement an innovation process in the maintenance management system. The procedure allows obtaining a knowledge base of the company's technical system of the present maintenance management. The proposal is composed by an audit and an expert analysis based on rules that include aspects related to the management form, the technological level of the productive equipment, the degree of organizational maturity, the maintenance goals and the physical and financial requirements to implement the selected conceptions. This procedure allows identifying the weaknesses and strengths of the productive system and the maintenance to plan in optimum form the proposed innovation maintenance.
\end{abstract}

Keywords: maintenance management, maturity analysis, maintenance conception 


\section{INTRODUCCIÓN}

Dentro de las funciones operativas tienen una alta relevancia producción y mantenimiento, ya que ambas tienen que mantener funcionando y mejorando la infraestructura productiva de la organización. Este tema refleja la importancia del mantenimiento en la estructura estratégica de las empresas (Muller et al., 2007; Kardec y Nacif, 2003; Swanson, 2001; Teixeira, 2001).

La innovación de la gestión del mantenimiento es un proceso sistemático, planeado, gerenciado, ejecutado y acompañado bajo el liderazgo de la alta administración, involucrando y comprometiendo a todos los gerentes, responsables y personal operacional de la organización (Arantes, 2002). El punto de inicio es una evaluación de la importancia de la función mantenimiento dentro de la empresa, y debe ser analizada en confrontación con los requisitos que son impuestos para atender el conjunto de equipos de acuerdo con el nivel de confiabilidad requerido. Esto debe conducir a la definición de la concepción del mantenimiento.

En la definición de la concepción del mantenimiento, está reflejado lo que la empresa espera que realice la función mantenimiento para acompañar a la consecución de sus objetivos de negocios, o sea debe ser direccionado (Waeyenbergh y Pintelon, 2007), lo que a su vez define los objetivos de la función. Esta es la base de todo el proceso de definición de la forma de la gestión del mantenimiento.

La innovación de la concepción del mantenimiento en las empresas es un proceso de aprendizaje por medio del cual se genera un flujo de nuevos conocimientos, competencias y capacidades. El conocimiento acumulado es el capital que posee la organización en el momento de enfrentar el desafío de implementar una nueva concepción (Forcadell y Guadamillas, 2002; Johannsen, 2000).

Diversas formas de concepción han sido propuestas y desarrolladas y sus aplicaciones son variadas, y las incluidas en este procedimiento son: Mantenimiento Centrado en la Confiabilidad (Carretero et al, 2003; Fleming y Oliveira, 1997), Mantenimiento Productivo Total (Tsuchiya, 1992), Mantenimiento Basado en el Riesgo (Khan y Haddara, 2003), Tero-Tecnología Avanzada (Sherwin, 2000), Concepción Estratégica del Mantenimiento (Murthy et al., 2002), Mantenimiento Centrado en el Negocio (Waeyenberg y Pintelon, 2002), Apoyo Logístico Integrado (Waeyenberg y Pintelon, 2002) y Mantenimiento con Calidad Total (Sherwin, 2000; Al-Najjar, 1996).

\section{METODOLOGIA}

Distintos autores mencionan que en un proceso de innovación es necesario tener en mente las características organizacionales presentes en la empresa como también las características propias del proceso de innovación (Nieto, 2004; Pratali, 2003; Chen y Small, 1996). Todo esto por el hecho de que muchas veces actúan en forma negativa factores como: no tener en cuenta las variables relacionadas con la preparación organizacional, no conocer la dinámica del proceso de innovación o no proyectar adecuadamente el proceso para conseguir unir fuerzas y apoyo.

En la elaboración del procedimiento se hace uso de una auditoria para simplificar la cuantificación de las variables definidas como importantes (Papazoglou et al., 2003), que en este caso es el proceso de innovación. El procedimiento entrega un análisis de la situación actual de la organización para el mantenimiento, como también para sus proyecciones futuras tomando como referencia la capacidad actual, todo basado en las respuestas que provee el responsable por el mantenimiento. El conocimiento dinámico es modelado por un procedimiento general de resolución en el cual cada función o paso está compuesto por un conjunto de reglas (Chtourou et al. 2005). El análisis de los resultados obtenidos por medio de la aplicación de las reglas, las cuales son definidas con un objetivo, permite sugerir acciones a implementar ya que todo el análisis está basado en el conocimiento que un especialista posee (Balachandra, 2000).

Se dio especial énfasis en la confección del procedimiento, en primer lugar, a la claridad para la captura de los datos y la entrega de la información, y en segundo lugar, la profundidad para poder identificar la realidad, las potencialidades y debilidades presentes en la gestión actual, además de las concepciones con más posibilidades de poner en práctica. Otros atributos importantes están relacionados con la amplitud, o sea, si el procedimiento cubre los suficientes aspectos que están presentes en la gestión del mantenimiento, y la sinergia, o sea, la capacidad de visualizar otros 
aspectos no cuantificables como ser estimular el dialogo, aunar conocimientos, capturar actitudes de liderazgo, etc. El procedimiento fue aplicado en cuatro empresas manufactureras con alto nivel de exportación de sus productos, en las cuales el mantenimiento tiene un rol importante y están procurando mejoras en su gestión para acompañar el desarrollo de la empresa.

En la aplicación del procedimiento se destacaron los siguientes aspectos: permite al sector del mantenimiento conocer el nivel de actualización de las herramientas de gestión que ellos están usando y las que se podrían implementar. Por otro lado da respaldo para hacer una síntesis del conocimiento que no se consigue tener en los cursos especializados que se venden como producto (una forma de concepción), y para el equipo de mantenimiento el procedimiento clarifica y apunta a las prácticas de la gestión, con destaque en la deficiencia y donde ella está, y para la fortaleza lo que debe ser hecho para mantenerla. Además, todos los grupos coincidieron en que la herramienta presentada a pesar de ser densa en su contenido entrega una evaluación muy certera de la realidad del mantenimiento en la empresa, destaca sus objetivos y da pautas claras sobre la forma que se debe seguir para realizar una innovación en la forma de gestión del mantenimiento.

Las dificultades detectadas en la aplicación dicen relación con la necesidad de realizar una capacitación previa sobre los conceptos de gestión del mantenimiento para los mantenedores y obtener un conocimiento acabado del nivel tecnológico y estructura productiva de la empresa por parte del facilitador del estudio. Aquí se revela la importancia del facilitador: tiene que ser un profesional que se involucre completamente con el personal de mantenimiento y los aspectos tecnológicos de la empresa, conocerlos en todas las dimensiones que el procedimiento necesita.

\section{EL PROCESO DE APLICACIÓN DEL PROCEDIMIENTO}

El objetivo de este procedimiento es entregar diagnósticos que orienten al líder del proyecto en la selección de la concepción de gestión para el mantenimiento, pero, con un conocimiento bastante preciso de su situación actual y la proyección en el futuro con más probabilidad de éxito. El producto final que el procedimiento propuesto ofrece al analista o administrador del mantenimiento es un conjunto de informaciones que apoyan su toma de decisiones. Este conjunto hace referencia a múltiples aspectos que son relevantes para la gestión del mantenimiento y que abarcan aspectos desde la madurez de la organización hasta una evaluación económica para las alternativas seleccionadas, ya que definir la mejor concepción del mantenimiento no es un problema trivial, porque las variables a ser consideradas varían de acuerdo con las empresas, tiempos, analistas y visiones gerenciales y donde un modelamiento matemático es extremadamente complicado.

\section{Módulo identificación y caracterización de la empresa}

En este módulo se obtienen los antecedentes básicos de la empresa los cuales son importantes porque sirven para tener referencias si se desea comparar formas de gestión entre empresas, como también son datos necesarios para tener un punto de inicio en la aplicación de la metodología. El informe emitido en esta primera recopilación de antecedentes está dividido en dos partes: la primera da un resumen de las características de la empresa y en la segunda parte se entrega un análisis que relaciona estas características con el mantenimiento, además de recomendaciones y críticas a los antecedentes introducidos, si estos contienen algunas incoherencias (Fig. 1).

\section{Modulo nivel del grado de atención a los requisitos}

En este módulo se explora la condición de los equipamientos para atender los requerimientos de negocio de la empresa, en conjunto con los servicios relacionados de mantenimiento, operaciones, abastecimiento y capacidad administrativa (Fig. 2). Los puntos incluidos son: 1) evolución del producto en el mercado: seguir la evolución tecnológica, estructural y operativa; 2) resultados económicos esperados: contribuir a la reducción de costos, al aumento del facturamiento, al retorno de la inversión o contribuir a la relación costo/beneficio; 3) productividad de los equipamientos: aumentar o mantener la productividad, 4) calidad de la producción: disminuir o mantener la tasa de rechazo; 5) flujos de masa y energía: atender los tiempos y volúmenes de entrega y los consumos de energía; 6) reglamentación ambiental: disminuir o mantener los niveles de polución, disminuir o mantener los niveles de vibraciones y 7) requisitos de seguridad: aumentar o mantener la seguridad del operador y del equipamiento. 
En el informe que se obtiene en este análisis están los antecedentes para que el administrador tome su primera decisión: ¿el grado de atención a los requerimientos de los recursos es el adecuado para enfrentar los desafíos de la empresa en relación a la evolución que tiene el producto en el mercado, teniendo presente el tiempo que toma implementar una concepción del mantenimiento?

\begin{tabular}{|r|r|}
\hline Informe de la identificación y caracterización de la empresa \\
Tipo de empresa: & Empresa de alimentos y vestuario \\
Actividad principal: & Fabricación de productos alimenticios \\
Tamaño de la empresa: & Empresa mediana: 100 a 499 funcionarios \\
Relación operador de producción por equipamiento: & Renor que 5 años \\
Grado de automatización mayor que 1: empresa con uso extensivo de mano de obra \\
Tipo de mantenimiento aplicado: & Con más de un operario por máquina \\
Para definir el tipo de manteniento preventivo: & Correctivo y preventivo según un programa \\
Caracterización relación producto/operación de la empresa: & Criterio definido por la empresa \\
\hline
\end{tabular}

\section{Percepción global sobre la forma de realizar el mantenimiento}

1.- La primera percepción global, basada en los parámetros identificados dan resultados coherentes con el tipo de gestión practicada. Antes de pasar al análisis del nivel de atendimiento, leer el resto de los comentarios.

2.- Para equipos con edad menor que 5 años, la operación debe ser controlada principalmente en forma automática, está bien direccionada la operación y la tarea del mantenimiento es mejorar este aspecto.

3.- Un buen mantenimiento debe apuntar a tener un equilibrio entre las acciones correctivas y las preventivas, dentro de un marco financiero que cumpla con los objetivos de negocio de la empresa, ¿es este su caso, ya que declaró tener ambos tipos?

4.- ¿Se ha medido la efectividad de los criterios impuestos para definir el tipo de mantenimiento a los equipos de la empresa?Comprobar este hecho es importante para una buena gestión.

5.- Si el equipamiento es automatizado es difícil tener a su alrededor más de un operario, revisar los datos o bien dar la razón del porqué. Podría ser usado este operario para prestar una ayuda importante en el proceso de mantenimiento.

Fig. 1: Informe sobre la caracterización de la empresa

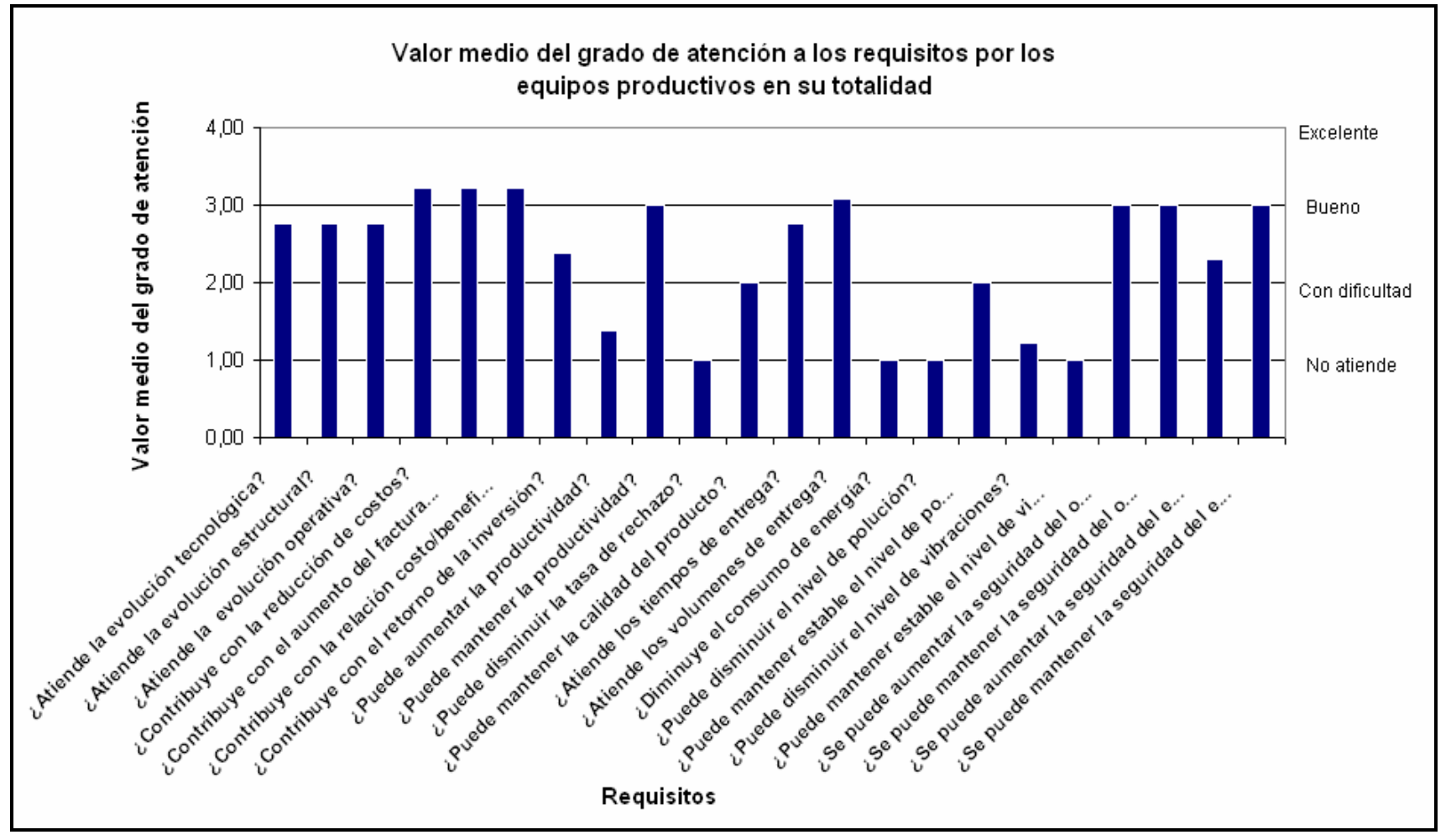

Fig. 2: Gráfico con el valor medio del grado de atención de cada requerimiento

\section{Módulo análisis detallado de la gestión actual}

Esta parte del procedimiento está orientada a evaluar a la función mantenimiento en relación a la gestión de los aspectos humanos, técnicos y económicos. La diferencia con el módulo anterior es 
que en este análisis la información es cruzada con las funciones típicas de la gestión y no se concentra en la parte técnica solamente (Fig. 3). La situación es analizada bajo el punto de vista de la capacidad administrativa del responsable del mantenimiento.

Los ítems de este módulo se refieren a: 1) aspectos técnicos: servicios, calidad de los servicios, métodos de trabajo, recursos, materiales y control de las actividades del mantenimiento; 2) aspectos humanos: relaciones internas, relaciones externas y organización de la función mantenimiento; 3) aspectos económicos: estructura y economía del mantenimiento y economía de la producción y 4) funciones administrativas: planeamiento, organización, análisis, ejecución e control. Para tener una alta probabilidad de éxito en la implementación de la innovación de la gestión, poseer infraestructura, recursos humanos capacitados y un ordenamiento administrativo, es su base fundamental.

\section{Módulo análisis de la madurez de la organización}

La madurez de la función indica que la organización definida para el mantenimiento está perfectamente condicionada para emprender todos sus proyectos de innovación, sean tecnológicos o de gestión integral de sus recursos. De acuerdo con Clarke y Garside (1997), en este procedimiento se incluirán los siguientes aspectos: compromiso con la implementación, cultura para enfrentar cambios, comunicación interna y externa, gestión de los conflictos, conocimiento y aplicación de herramientas y metodologías de proyecto (Fig. 4).

La madurez es principalmente, la combinación del apoyo o comprometimiento que el entorno le puede ofrecer a la función mantenimiento, en especial de los niveles superiores de la empresa, más la capacidad o conocimiento que se posee de las metodologías relacionadas con la implementación de proyectos, como también tienen una gran influencia la voluntad de la organización en involucrarse en proyectos nuevos, la cantidad y calidad de la información disponible y finalmente la capacidad de solucionar conflictos producto del acceso a los recursos de la empresa.

En este punto de la aplicación del procedimiento el administrador decide nuevamente: ¿el nivel de madurez de la organización es el adecuado para enfrentar el proceso de innovación?

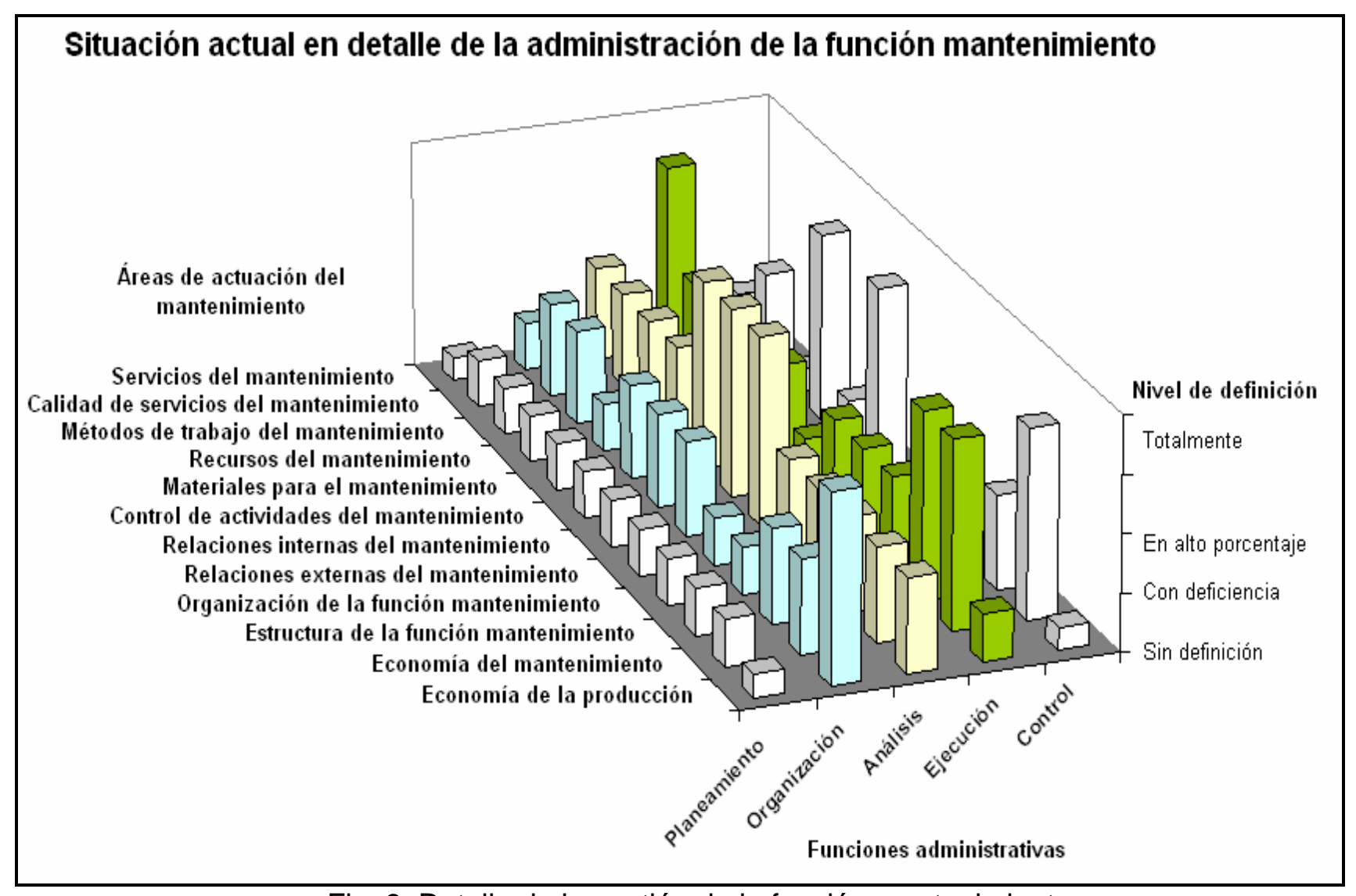

Fig. 3: Detalle de la gestión de la función mantenimiento 


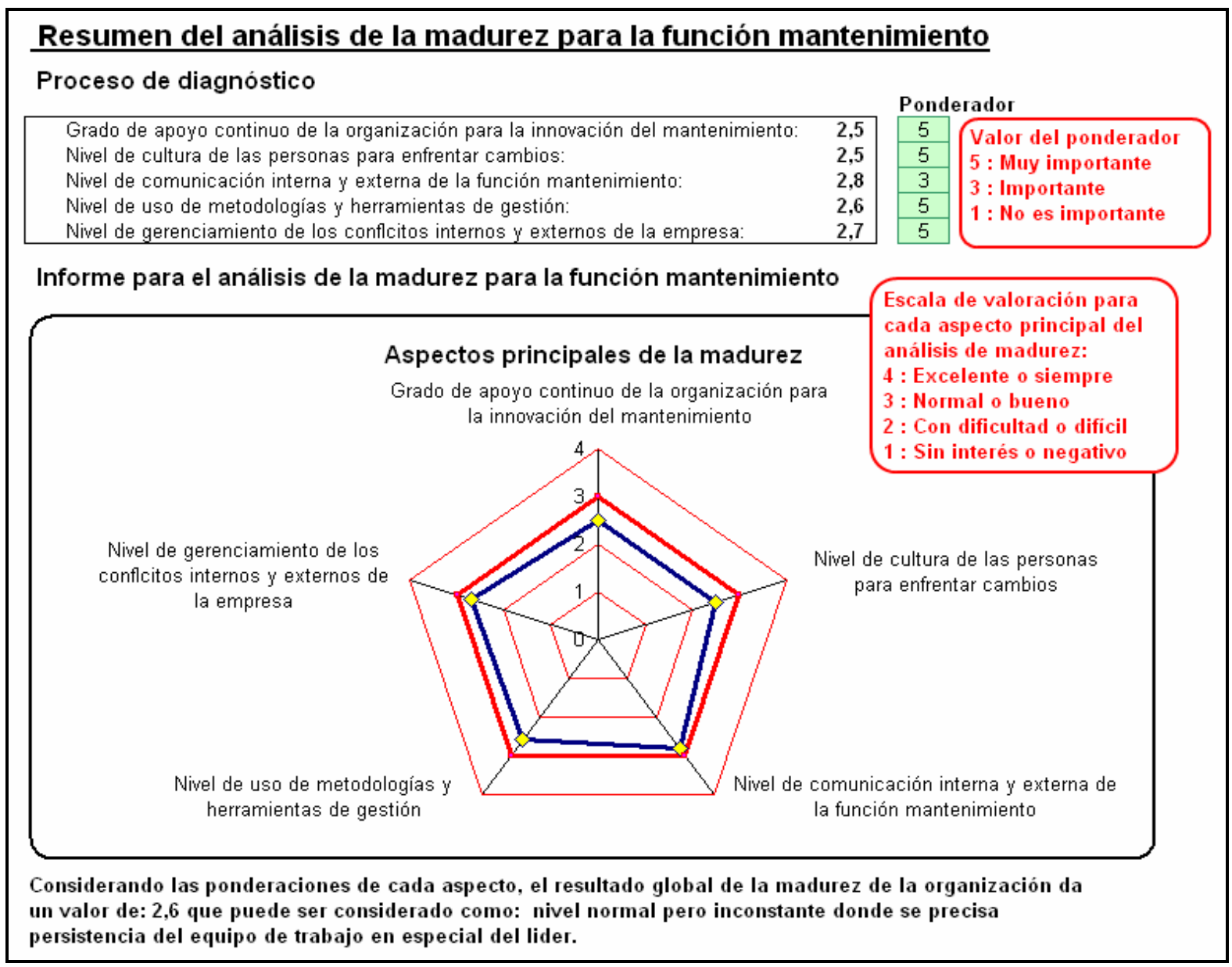

Fig. 4: Resumen con todos los conceptos de la madurez para la organización

\section{Módulo definición de los parámetros de selección de la concepción del mantenimiento}

En esta parte del proceso de selección de la concepción del mantenimiento se reúnen todos los datos introducidos hasta este punto del análisis y son contrastados con los requisitos que exigen cada concepción y su campo de aplicabilidad, a fin de recomendar un conjunto de ellas que mejor respondan a las metas de la función mantenimiento. Esta selección se hace en base a los parámetros que el facilitador define de acuerdo con su apreciación sobre las condicionantes de la empresa, los requisitos actuales que son impuestos, el tipo de mercado que enfrenta y las capacidades que posee la función mantenimiento.

El administrador, además, debe evaluar sus capacidades y complementar el análisis implementado en el programa para seleccionar de forma más adecuada, de acuerdo a sus posibilidades, un conjunto de concepciones. Para tal efecto se identifican para cada concepción los requisitos de información y conocimientos, de técnicas y herramientas, de apoyo y logística y de infraestructura. Para cada aspecto, el administrador debe indicar cuales de estos requisitos está en condiciones de poner en práctica inmediatamente. El ideal es que la selección de las concepciones esté basada en aquellas condiciones en las cuales se tienen las más altas capacidades (Fig. 5).

\section{Módulo análisis económico de las alternativas seleccionadas}

La evaluación económica es un paso importante en la selección final de la concepción ya que en esta parte se valora el real impacto de la innovación en la gestión. La ganancia adicional de este análisis es que obliga al equipo de trabajo a investigar y evaluar en profundidad sobre los costos y beneficios relacionados con el mejoramiento de las capacidades instaladas, y sobre las que pueden acceder dentro o fuera de la organización, para así tener valores de acuerdo con su realidad. El resultado de este módulo es el beneficio neto presente y la tasa interna de retorno. Esta última información debe ser comparada con la tasa exigida por la empresa para los proyectos de inversión y 
se indica cual de las concepciones cumple con la condición financiera. Si no existen condicionantes externas, la concepción que resulte en la mayor tasa debería ser implementada.

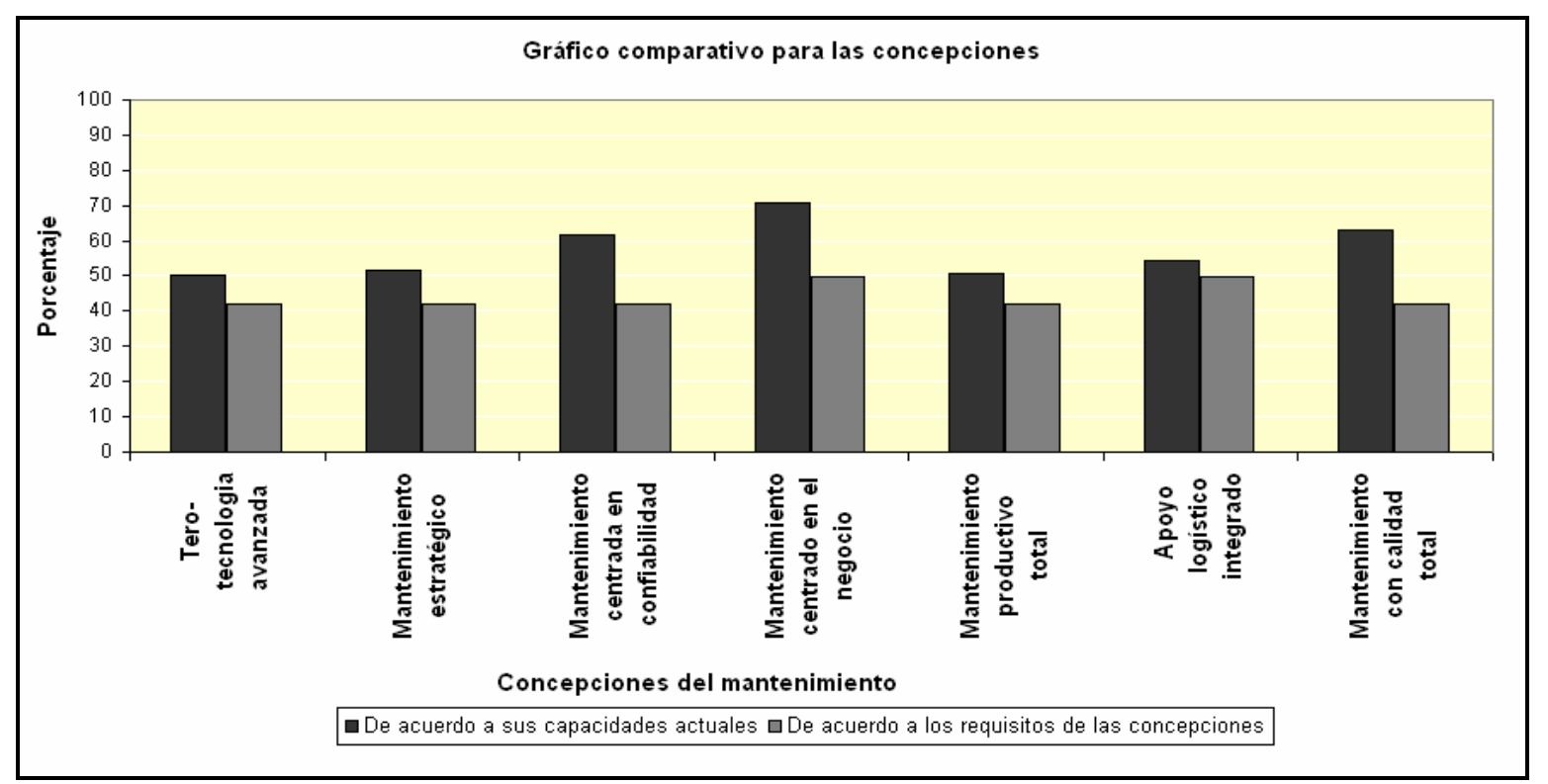

Fig. 5: Gráfico para las concepciones de mantenimiento analizadas

En la metodología se propone hacer la evaluación económica para dos horizontes de planeamiento: 2 y 4 años, todo en relación a la estructura de actual costos directos e indirectos de mantenimiento de la empresa. Se proponen estos horizontes para el análisis económico porque en la práctica el período medio para implementar un proceso de innovación varia entre un año y medio y cuatro años (Chan et al., 2005; Rausand, 1998; Chen y Small, 1996).

\section{CONCLUSIÓN}

El procedimiento presentado para apoyar el proceso de innovación de la gestión del mantenimiento nació de la necesidad de dotar al tomador de decisiones del área, de una herramienta que le permitiera evaluar sus condiciones para adoptar una nueva forma de concepción del mantenimiento y de esta forma elaborar un proyecto real para sus necesidades de innovación. En la aplicación los integrantes del equipo de mantenimiento pudieron evaluar en primera instancia lo siguiente: sus necesidades de preparación en herramientas y metodologías de proyectos, la percepción del entorno de la función mantenimiento en la empresa, las potencialidades de los recursos disponibles, la compatibilización de sus metas con la de la empresa, adaptabilidad de concepciones a distintas áreas de actuación y el volumen de trabajo que implica la implementación de un proceso de innovación, aspectos que deben ser dimensionados antes de emprender un proyecto de innovación. O sea, este procedimiento le revela al equipo humano, a cargo del mantenimiento, su condición actual y cual es la tarea que le espera al frente, para alcanzar las metas que declararon como propias de la función a fin de atender los requerimientos que le impone el negocio de la empresa.

\section{REFERENCIAS}

Al-Najjar, B.; Total quality maintenance. An approach for continuous reduction in costs of quality products. Journal of Quality in Maintenance Engineering, 2(3), 4-20 (1996).

Arantes Da Costa; E. Gestão estratégica, Editora Saraiva (2002).

Balachandra, R.; An expert system for new product development projects. Industrial Management \& Data Systems 100/7, 317-324 (2000).

Carretero, J. y otros nueve autores; Applying RCM in large scale systems: a case study with railway networks. Reliability Engineering and System Safety 82, 257-273 (2003).

Chan, F.T.S. y otros cautro autores; Implementation of total productive maintenance: a case study. International Journal of production Economics, 95(1) 71-94 (2005). 
Chen, I. y M. Small; Planning for advanced manufacturing technology. International Journal Of Operation \& Production Management, 16(5), 4-24 (1996).

Chtourou, H., W. Masmoudi y A. Maalej; An expert system for manufacturing systems machine selection. Expert Systems with Applications 28, 461-467 (2005).

Clarke, A. y J. Garside; The development of a best practice model for change management. European Management Journal, 15(5) (1997).

Fleming, P.V. y F. Oliveira; Considerações sobre a implementação conjunta de TPM e MCC na indústria de processos. 12o Congresso Brasileiro de Manutenção da ABRAMAN, outubro de 1997, São Paulo-SP (1997).

Forcadell, F. y F. Guadamillas; A case study on the implementation of a knowledge management. Strategy Oriented to Innovation Knowledge and Process Management, 9(3), 162-171, (2002).

Johannsen, C.G; Total quality management in a knowledge management perspective. Journal of Documentation, 56(1) (2000).

Kardec, A. y J. Nascif; Manutenção: Função Estratégica. Ed. Qualitymark (2003).

Khan, F.I. y M.M. Haddara; Risk-based maintenance (RBM): a quantitative approach for maintenance/inspection scheduling and planning. Journal of Loss Prevention in the Process Industries No 16, 561-573 (2003).

Muller, A., M.C. Suhner y B. Lung; Formalisation of a new prognosis model for supporting proactive maintenance implementation on industrial system. Reliability Engineering and System Safety (2007), doi:10.1016/j.ress.2006.12.004

Murthy, D.N.P., A. Atrens y J.A. Eccleston; Strategic maintenance management. Journal of Quality in Maintenance Engineering, 8(4) (2002).

Nieto, M.; Basic propositions for the study of the technological innovation process in the firm. European Journal of Innovation Management, 7(4), 314-324 (2004).

Papazoglou, I.A. y otros seis autores; I-Risk: development of an integrated technical and management risk methodology for chemical installations. Journal of Loss Prevention in the Process Industries 16, 575-591 (2003)

Pratali, P.; Strategic management of technological innovations in the small to medium enterprise. European Journal of Innovation Management, 6(1), 18-31 (2003).

Rausand, M; Reliability centered maintenance. Reliability Engineering and System Safety, 60 (1998).

Sherwin, D.; A review of overall models for maintenance management. Journal of Quality in Maintenance Engineering, 6(3), 138-164 (2000).

Swanson, L.; Linking maintenance strategies to performance. Int. J. Production Economics, 70 (2001).

Teixeira, A.; Multicriteria decision on maintenance: spares and contract planning. European Journal of Operational Research, 129 (2001).

Tsuchiya, S.; Quality maintenance: zero defects through equipment management. Productivity Press, Cambridge, MA, p.4, (1992).

Waeyenbergh, G. y L. Pintelon; A framework for maintenance concept development. International Journal of Production Economics 77, 299-313 (2002)

Waeyenbergh, G. y L. Pintelon; CIBOCOF: A framework for industrial maintenance concept development, International Journal of Production Economics (2007), doi:10.1016/j.ijpe.2006.10.012 